\title{
Efficient Entanglement of Spin Qubits Mediated by a Hot Mechanical Oscillator
}

\author{
Emma Rosenfeld, ${ }^{1, *}$ Ralf Riedinger@ ${ }^{1}$ Jan Gieseler@ ${ }^{1, \dagger}$ Martin Schuetz, ${ }^{2,3,}$ and Mikhail D. Lukin ${ }^{1}$ \\ ${ }^{1}$ Physics Department, Harvard University, Cambridge, Massachusetts 02318, USA \\ ${ }^{2}$ Amazon Quantum Solutions Lab, Seattle, Washington, D.C. 98170, USA \\ ${ }^{3}$ AWS Center for Quantum Computing, Pasadena, California 91125, USA
}

(Received 5 November 2020; accepted 29 April 2021; published 24 June 2021)

\begin{abstract}
Localized electronic and nuclear spin qubits in the solid state constitute a promising platform for storage and manipulation of quantum information, even at room temperature. However, the development of scalable systems requires the ability to entangle distant spins, which remains a challenge today. We propose and analyze an efficient, heralded scheme that employs a parity measurement in a decoherence free subspace to enable fast and robust entanglement generation between distant spin qubits mediated by a hot mechanical oscillator. We find that high-fidelity entanglement at cryogenic and even ambient temperatures is feasible with realistic parameters and show that the entangled pair can be subsequently leveraged for deterministic controlled-NOT operations between nuclear spins. Our results open the door for novel quantum processing architectures for a wide variety of solid-state spin qubits.
\end{abstract}

DOI: 10.1103/PhysRevLett.126.250505

Introduction.-Electronic and nuclear spin qubits in the solid state are encouraging candidates for the realization of quantum information systems. Over the past decade, longlived quantum memories and few-qubit registers have been demonstrated in several different platforms, including under ambient conditions. The key outstanding challenge is engineering fast, programmable interactions between spin qubits separated by micrometer-scale distances. For example, color centers such as the nitrogen vacancy (NV) center in diamond are promising contenders as robust qubits, owing to their long coherence times at room temperature [1], well-developed microwave control, and optical initialization and readout. However, generating entanglement on demand between spins remains a challenge: the short-range dipole-dipole interaction limits connectivity [2], while optical entanglement schemes are inefficient [3-5], require cryogenic temperatures, and decohere nuclear memories [6,7].

In a complementary approach, Rabl et al. [8] suggested transducing interactions via magnetically functionalized oscillators, leveraging recent advances in the control of micromechanical resonators $[9,10]$, which enables quantum control of solid-state electron spins [11-15]. Robustness against thermal noise is desirable for such applications at elevated temperatures to avoid phononinduced gate errors [16]. Previous approaches for such "hot" gates require large qubit-resonator cooperativities $C \gg 1$ for low error rates [17] with error scaling as $\mathcal{E} \propto 1 / \sqrt{C}\left(C \equiv \lambda^{2} / \Gamma \kappa n_{\text {th }}\right.$ compares the coherent coupling rate $\lambda$ to the dissipation rates of the spin and resonator, $\Gamma$ and $\kappa n_{\text {th }}$, respectively). These regimes are experimentally challenging to achieve, such that a demonstration of mechanically mediated entanglement remains elusive.
In this Letter, we propose and analyze a fast and robust entanglement protocol for two spins (with eigenstates $|0\rangle$ and $|1\rangle)$, linearly coupled to a common mode of a hightemperature mechanical resonator [Fig 1(a)] via parity (a)

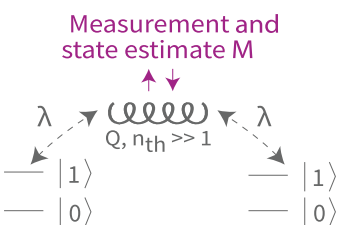

(c)

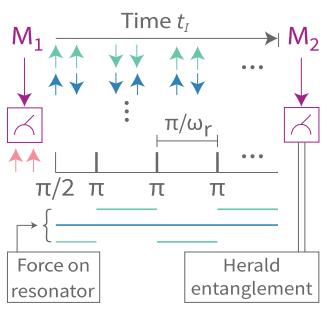

(b)

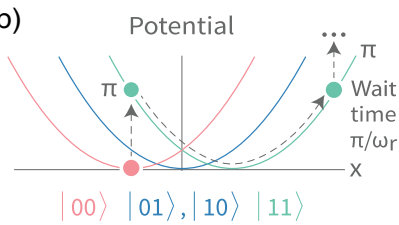

(d)

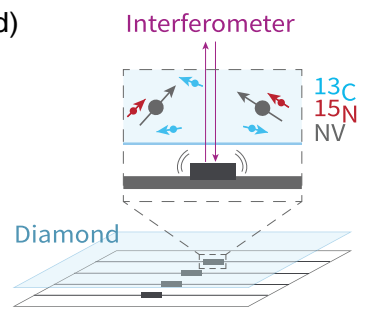

FIG. 1. Entanglement protocol. (a) Two qubits are coupled with equal strength $\lambda$ to a high $Q$ resonator that is independently measured and has the thermal occupation $n_{\text {th }} \gg 1$. (b) Spindependent resonator potential. Spin states $|01\rangle,|10\rangle$ are decoupled, whereas $|00\rangle,|11\rangle$ shift the potential, such that toggling between them every half period drives the resonator. (c) The spins are initialized in $|++\rangle$, and the resonator states before $\left(M_{1}\right)$ and after $\left(M_{2}\right)$ applying the spin pulse sequence (black) for duration $t_{I}$, are compared. Absence of displacement indicates spins are in the entangled antiparallel states. (d) Proposed implementation. A diamond with NV centers is placed near a microresonator (gray) functionalized with nanomagnets (black), which is measured interferometrically. The entangled $\mathrm{NV}$ spin state is used to teleport gates between proximal ${ }^{13} \mathrm{C}$ or ${ }^{15} \mathrm{~N}$ nuclear spins. 
measurements in a decoherence-free subspace (DFS) [18-21]. The Bell states $\left|\Psi^{ \pm}\right\rangle \propto|01\rangle \pm|10\rangle$ decouple from the resonator, forming a DFS insensitive to its thermal fluctuations. In contrast, the aligned states $|00\rangle$ and $|11\rangle$ exert a force on the oscillator, resulting in a large, observable displacement [Fig 1(b)]. We can thus herald the entangled $\left|\Psi^{ \pm}\right\rangle$state by monitoring the absence of an excess force on the mechanical oscillator, constituting a (half) parity measurement in a measurement-free subspace $[19,20,22]$ analogous to previous proposals considering atoms in optical cavities [18]. By design, this protocol is robust to thermal noise and does not require strong coupling or cooling to the mechanical ground state. We show that entanglement can be generated at high success rates with relaxed cooperativity requirements, $C \gtrsim 1$, and with error scaling approaching $\mathcal{E} \propto \ln (C) / C$ at large cooperativities. We specifically analyze an experimental realization involving $\mathrm{NV}$ centers in diamond coupled to magnetically functionalized mechanical nanobeam resonators $[12,13,15,23]$, but note that our protocol can equally be applied to other qubit species coupled to bosonic modes at high temperatures [24-34], even when high-fidelity readout is not available by other means [11,35-37].

The entangled pair of electronic spins can be subsequently leveraged to herald two-qubit gates between nearby, coherently coupled nuclear spin memories in the solid state [Fig 1(d)]. Assuming state-of-the-art quality $(Q)$ factors, spin-mechanics coupling strength, and spin coherence times [9,38-41], we expect that our entangling gate can achieve error rates below $1 \%$ at cryogenic temperatures. With modest improvements in the coupling strength, similarly low error rates can be achieved at room temperature.

Entanglement protocol.-The key idea of our approach can be understood by considering two spin qubits, characterized by the Pauli operators $\sigma_{x, y, z}^{(i)}(i=1,2)$, that are linearly coupled with equal strength $\lambda$ to a micromechanical oscillator [Fig. 1(a); see the Supplemental Material (SM) [42], which contains Refs. [43-53], for inhomogeneous $\lambda$ ). If the qubit frequencies $\omega_{s}^{(i)}(i=1,2)$ strongly exceed that of the resonator $\omega_{r} \ll \omega_{s}^{(i)}$, the transverse coupling terms can be ignored, and the system Hamiltonian is

$\mathcal{H} / \hbar=\frac{\omega_{s}^{(1)}}{2} \sigma_{z}^{(1)}+\frac{\omega_{s}^{(2)}}{2} \sigma_{z}^{(2)}+\omega_{r} a^{\dagger} a+\lambda S_{z}\left(a+a^{\dagger}\right)$,

where $S_{z}=\sigma_{z}^{(1)}+\sigma_{z}^{(2)}$ and $a\left(a^{\dagger}\right)$ are the bosonic annihilation (creation) operators of the resonator mode. For the two states $|01\rangle$ and $|10\rangle$, the qubits are decoupled from the resonator: the $S_{z}=0$ states comprise a DFS, i.e., their phase is independent of the mechanical state. The other two states exert a force $\sim \pm 2 \hbar \lambda / z_{p}$ on the resonator, where $z_{p}$ is the mechanical zero point fluctuation.
In our entanglement protocol [Fig. 1(c)], (i), the state of the resonator is first measured, while the two spins are initialized in the separable state $|+\rangle \otimes|+\rangle \propto \sqrt{2}\left|\Psi^{+}\right\rangle+$ $|11\rangle+|00\rangle$, with $|+\rangle=(|1\rangle+|0\rangle) / \sqrt{2}$. Then, (ii) the spins interact with the resonator for a time $t_{I}$ while being subjected to a special resonant decoupling sequence such that the spin states $|11\rangle$ and $|00\rangle$ displace the resonator state. Finally, (iii) the resonator displacement is measured. If it is below a threshold, the spins are projected into the Bell state $\left|\Psi^{+}\right\rangle$, indicating successful entanglement generation. The protocol can be made deterministic by repeating steps (i)-(iii) until success $(\sim 2-3$ repetitions in the regime of interest).

To estimate its practical performance, we assume the mechanical system can be described by a master equation and is weakly coupled $(Q \gg 1)$ to a hot thermal bath at rate $\kappa=\omega_{r} / Q$ and temperature $T \gg \hbar \omega_{r} / k_{B}$. We also assume each qubit is dispersively coupled to an independent reservoir with dephasing rate $\Gamma$. The Gaussian state of the oscillator can be estimated interferometrically and independently of the spins. In practice, the effects of the measurement backaction and duration are negligible in near term realizations (see the SM). To simplify the derivation, in the following we assume short backaction evading measurements of the mechanical quadrature [54], neglecting the measurement duration and avoiding a lower limit on the measurement uncertainty.

Figure 2 illustrates the key ingredients of the scheme. In step (i), we perform an initial linear measurement $M_{1}$ on the momentum quadrature $p \equiv i\left(a^{\dagger}-a\right) / \sqrt{2}$, with measurement uncertainty $\Delta m$ [Fig. 2(a)]. In step (ii), the spins are resonantly coupled to the oscillator by a series of $\pi$ pulses (here assumed to be ideal), with a pulse separation $2 \tau=\pi / \omega_{r}$. This simultaneously maximizes the conditional mechanical displacement and the spin coherence by dynamically decoupling from their bath [Figs. 1(b) and 1(c)]. Throughout this pulse sequence, the force acting on the resonator is a square wave with amplitude $\sim-\hbar \lambda S_{z} / z_{p}$ [Fig. 1(c)] and frequency $\omega_{r}$. In the high $Q$ limit, higher harmonics of the force can be neglected, resulting in the effective interaction Hamiltonian in the rotating and toggling frame under a rotating wave approximation [42]:

$$
\mathcal{H}_{\text {int }} / \hbar=\frac{2 \lambda}{\pi} S_{z}\left(a^{\dagger}+a\right),
$$

leading to a momentum shift of the resonator $\mu\left(S_{z}, t_{I}\right)=$ $-4 \sqrt{2} \lambda S_{z}\left(1-e^{-\kappa t_{I} / 2}\right) / \pi \kappa \approx-2 \sqrt{2} \lambda S_{z} t_{I} / \pi$ in natural units for $\kappa t_{I} \ll 1$. As the conditional equations of motion are linear, the motional states after step (ii) remain Gaussian with an uncertainty $\Delta d\left(t_{I}\right) \approx \sqrt{\kappa n_{\text {th }} t_{I}+\Delta m^{2}}$ [dashed circles in Fig. 2(b)] and a spin-dependent expectation value of the momentum quadrature of $M_{1} e^{-\kappa t_{I} / 2}+\mu\left(S_{z}, t_{I}\right)$. Then, (iii) a second measurement $M_{2}$ localizes the resonator with uncertainty $\Delta m$, projecting the spin population 


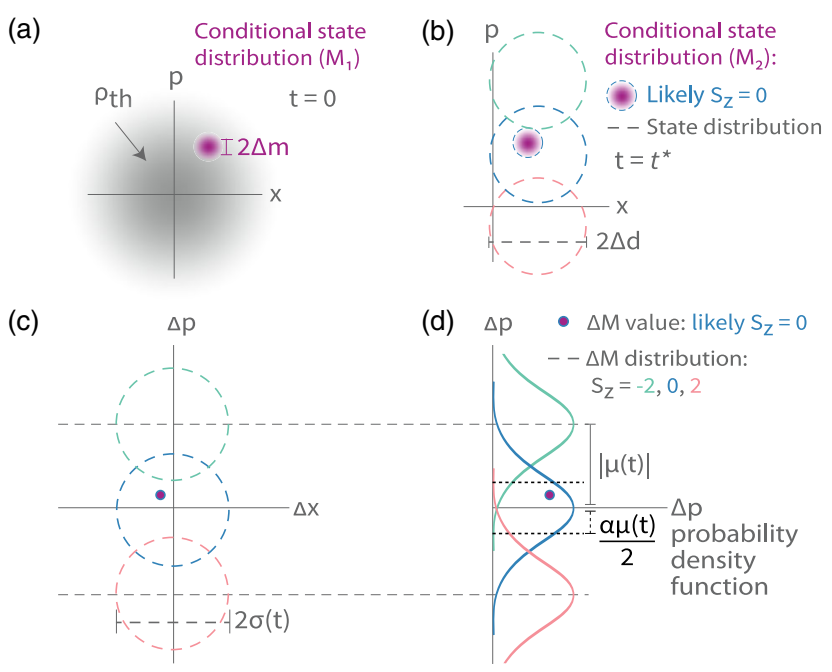

FIG. 2. Mechanical phase space picture. (a) The thermal resonator $\left(\rho_{\text {th }}\right)$ is localized by measurement $M_{1}$ (uncertainty $\Delta m$, purple) at time $t=0$ [55-58]. (b) After interaction time $t_{I}$, the spin-conditional resonator states (dashed circles, $S_{z}=2$ pink, $S_{z}=0$ blue, $S_{z}=-2$ green) separate, and the resonator is measured again $\left(M_{2}\right.$, purple). (c) The conditional distribution of measured displacement (dashed circles) and (d) its projection onto the momentum basis used to define a threshold $\alpha|\mu| / 2$ (black dashed line for $\alpha \approx 0.6$ ). If a specific measurement (purple dot) lies within this threshold, $\left\langle S_{z}\right\rangle=0$ is assigned and entanglement is heralded.

to $\left\langle S_{z}\right\rangle \in\{0,+2,-2\}$ if the distributions are separable. If this is achieved within the coherence time $1 / \Gamma$ of the spins, an $\left\langle S_{z}\right\rangle=0$ measurement projects the two spins into the entangled state $\left|\Psi^{+}\right\rangle$.

A simple estimate shows the minimum requirements for this protocol. For negligible measurement uncertainties $\Delta m^{2} \ll \kappa n_{\mathrm{th}} t_{I}$ and an interaction time comparable to the spin coherence time $t_{I} \sim 1 / \Gamma$ yet shorter than the mechanical lifetime $t_{I} \ll 1 / \kappa$, the distributions become separable if the displacement $|\mu(2,1 / \Gamma)| / 2$ exceeds the uncertainty $\Delta d(1 / \Gamma)$, i.e., $\lambda^{2} / \Gamma \kappa n_{\mathrm{th}}=C \gtrsim 1$.

To obtain an estimate of the fidelity, we compute the (Gaussian) probability density function $\mathcal{P}_{\left\langle S_{z}\right\rangle}(\Delta M)$ of the momentum difference $\Delta M \equiv M_{2}-e^{-\kappa t_{I} / 2} M_{1}$, conditional on the spin state $S_{z}$, which has expectation value $\mu\left(S_{z}, t_{I}\right)$ and variance

$$
\sigma\left(t_{I}\right)^{2}=\Delta m^{2}\left(1+e^{-\kappa t_{I}}\right)+n_{\mathrm{th}}\left(1-e^{-\kappa t_{I}}\right)
$$

corresponding to the contributions by the measurement uncertainties and diffusion during the interaction. The state is assumed to have $S_{z}=0$ if

$$
|\Delta M|<\alpha \mu\left(2, t_{I}\right) / 2,
$$

where the threshold $\alpha \in(0,1]$ can be tuned to trade between a high acceptance rate $(\alpha \rightarrow 1)$ and low false positive acceptance $(\alpha \rightarrow 0)$. The probability of an accepted event being a true positive is $S\left[\alpha, g\left(t_{I}\right)\right] \approx$ $1 /\left[1+e^{-2 g\left(t_{I}\right)^{2}}\right]-\mathcal{O}\left(\alpha^{2}\right)$, given by the integrals of $\mathcal{P}_{0, \pm 1}$ within the thresholds and weighted by the initial spin populations [42], where we define the normalized displacement $g\left(t_{I}\right) \equiv \mu\left(2, t_{I}\right) / 2 \sigma\left(t_{I}\right)$. The error in entanglement fidelity $\mathcal{F}=\left\langle\Psi^{ \pm}|\rho| \Psi^{ \pm}\right\rangle$is the result of two independent error sources, namely spin dephasing and false positive $\left\langle S_{z}\right\rangle=0$ assignments, yielding

$\mathcal{F}=\frac{1+e^{-2 \Gamma t_{I}}}{2} S\left[\alpha, g\left(t_{I}\right)\right] \approx \frac{1}{2} \frac{1+e^{-2 \Gamma t_{I}}}{1+e^{-2 g\left(t_{I}\right)^{2}}}-\mathcal{O}\left(\alpha^{2}\right)$.

It follows directly that entanglement can be generated $(\mathcal{F}>1 / 2)$ for $g\left(t_{I}\right)^{2}>\Gamma t_{I}$. We note that this simple estimate for $\alpha \rightarrow 0$ is a good approximation for general $\mathcal{F}[42]$.

Analysis of the Bell state preparation.-In the following, we consider the experimentally relevant regime $\Delta m^{2} \ll \kappa n_{\text {th }} t_{I}$, i.e., the linearized diffusion term dominates the variance of $\Delta M$ and that $\kappa t_{I} \ll 1$. In these limits,

$$
g\left(t_{I}\right)^{2} \approx\left(8 / \pi^{2}\right) C \Gamma t_{I}
$$

such that $\mathcal{F}$ can be described only in terms of $\alpha, C$, and $\Gamma t_{I}$ [Fig. 3(a)]. The fidelity exceeds $1 / 2$ for $C>\pi^{2} / 8 \sim 1.2$
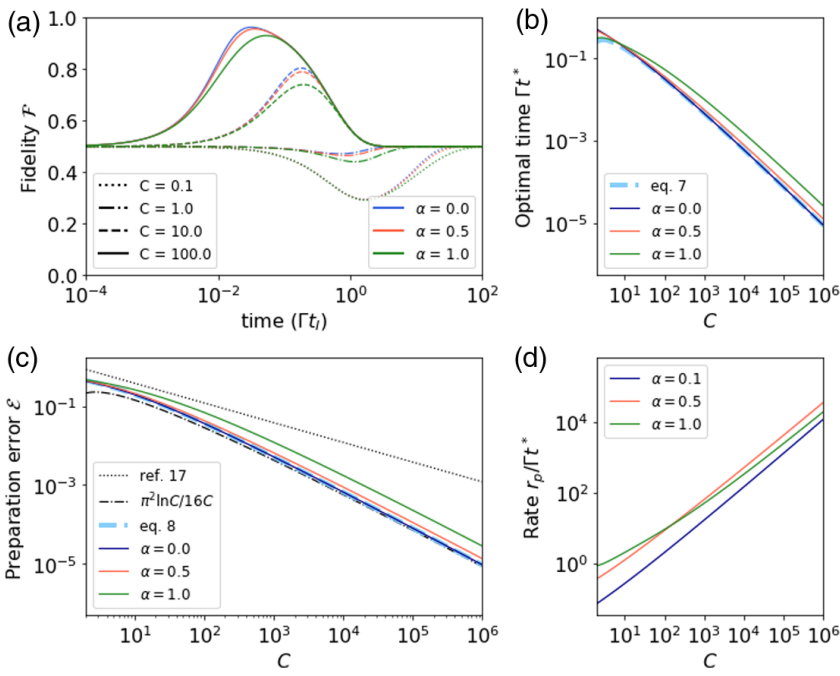

FIG. 3. Performance. (a) Fidelity $\mathcal{F}$ as a function of $\Gamma t_{I}$ under Eq. (6). Thresholds $\alpha$ are color coded and cooperativities $C$ have the associated line styles. Analytic form in Eq. (5), corresponding to $\alpha \ll 1$, blue curves. (b) Optimal pulse sequence duration (solid lines) and its analytic approximation [Eq. (7)] (dashed line). (c) Infidelity $\mathcal{E}$ of the Bell state preparation (solid lines) and analytic approximation [Eq. (8)] for $\alpha \ll 1$ (blue dashed line). The black dotted line represents the optimal infidelity of a deterministic hot gate [17], and the dashed-dotted black line is the asymptotic $\pi^{2} \ln C / 16 C$ scaling. (d) Normalized rate of true positive entanglement heralding events $r_{p} / \Gamma$. The fast repetition rate allows multiple protocol attempts within the spin coherence time for large $C$. 
and exceeds $96 \%$ for $C \sim 100$, demonstrating that our protocol can be applied with relatively low $C$. The optimal interaction time $\Gamma t^{*}$ is determined numerically for each $C$ [Fig. 3(b), dashed lines] and can be analytically approximated as

$$
\Gamma t^{*} \approx \frac{\pi^{2}}{16 C} \ln \left(16 C / \pi^{2}-1\right)
$$

for $C>\pi^{2} / 8$ and $\Delta m, \alpha \rightarrow 0$ [42], showing that the entanglement protocol is fast compared to the spin coherence time. We note that, in the regime of interest $(C \gtrsim 8$, $t_{I} \ll 1 / \Gamma$ ), decreasing the threshold $\alpha$, e.g., from 1 to 0.5 , reduces the optimal interaction time [Fig. 3(b)]. At high $C$, this can compensate the reduced acceptance rate for small $\alpha$ and increase the absolute rate of true positive entanglement heralding events given by $r_{p}=\int_{-\theta}^{+\theta} \mathcal{P}_{0}(p) d p / 2 t^{*}$ for threshold $\theta=\alpha \mu\left(+2, t^{*}\right) / 2$ [Fig. 3(d)] [42]. Inserting Eq. (7) into Eq. (5), we find a lower bound to the fidelity

$$
\mathcal{F} \geq \frac{1}{2} \frac{1+\left(16 C / \pi^{2}-1\right)^{-\pi^{2} / 8 C}}{1+\left(16 C / \pi^{2}-1\right)^{-1}}
$$

again for $C>\pi^{2} / 8$ and $\Delta m, \alpha \rightarrow 0$. The error $\mathcal{E}=1-\mathcal{F}$ is shown in Fig. 3(c). Remarkably, the cooperativity required to achieve an error $\mathcal{E}<10^{-3}$ is more than 2 orders of magnitude lower than for previous mechanically mediated gates [8,17]. For large $C, \mathcal{E} \sim\left(\pi^{2} / 16\right) \ln (C) / C$.

Potential applications. - The entanglement protocol presented here is inherently probabilistic, approaching a heralding probability of $1 / 2$ for $\alpha \rightarrow 1$. However, it can be extended to yield deterministic controlled-NOT gates, as required for quantum information processing, between associated qubit registers by feedback, assuming a simple repeat-until-success scheme $[4,18]$. In the following, we consider two electronic spins, such as NV centers (using $\left|m_{s}\right\rangle=| \pm 1\rangle$ as qubit states for maximal displacements), interacting with the mechanical resonator and coupled to nearby ${ }^{13} \mathrm{C}$ (or ${ }^{15} \mathrm{~N}$ ) nuclear spins in the diamond host [Fig. 1(d)]. The entangled NV spin state is used to teleport a gate between the nuclear spins [59]. Contributions to the gate error $\mathcal{E}_{T}$ include infidelities related to the ideal entanglement protocol $\mathcal{E}$, control $\left(\mathcal{E}_{C}\right)$, initialization $\left(\mathcal{E}_{\text {init }}\right)$, and readout $\left(\mathcal{E}_{\mathrm{RO}}\right)$ of the $\mathrm{NV}$ spins, and the electron-nuclear CNOT gate $\left(\mathcal{E}_{\mathrm{CNOT}}\right)$. Nuclear qubit errors arise from coupling to a bath $\left(\mathcal{E}_{\text {nuc }}\right)$ at rate $\Gamma \gamma_{N} / \gamma_{e}$, where $\gamma_{N}\left(\gamma_{e}\right)$ is the nuclear (electron) gyromagnetic ratio, as well as dephasing due to electron spin control errors in failed entanglement attempts. As the latter depends on the heralding probability and the hyperfine coupling, we attribute it to $\mathcal{E}_{C}$ with a factor $\eta$, which is below 1 in the regime of interest [42]. Combining state-of-the-art spin control $[60,61]$ with robust decoupling sequences [62], $\mathcal{E}_{C}$ can be neglected. However, if left unaddressed without optimal spin control, $\mathcal{E}_{C}$ can limit the fidelity [7]. As the repetition rate is high [Figs. 3(b) and 3(d)], we further neglect the small probability of failure after a large number of repetitions in a synchronous circuit [4]. In this case, the total error of the deterministic nuclear gate is $\mathcal{E}_{T}=\mathcal{E}+2\left[(1+\eta) \mathcal{E}_{C}+\mathcal{E}_{\text {init }}+\mathcal{E}_{\mathrm{RO}}+\mathcal{E}_{\mathrm{CNOT}}+\mathcal{E}_{\text {nuc }}\right]$.

Experimental implementation.-In NV-based operations on nearby nuclear spins, optical excitation of the NV induces decoherence on the nuclear spins through the hyperfine coupling $[6,7,63,64]$, which can limit $\mathcal{E}_{T}$ due to repeated spin initialization and readout unless compensated by a DFS of nuclear spins [65]. In our system, the mechanical oscillator can also be used for single-shot readout and initialization [11], eliminating the need for optical illumination of the color center [66].

While state-of-the-art continuous interferometric measurement schemes are sufficient for initial experiments $[38,40]$, their performance could be optimized using a Kalman filter [see, e.g., Fig. 2] [55-58,67] to account for mechanical spectator modes and other technical noise sources [42]. For further improvement, the estimation of the spin-induced displacement can be achieved with a multiple model adaptive estimation [68,69], while performing steps (ii) and (iii) simultaneously, as well as feedback on the spin state with additional global spin rotations during the pulse sequence, further increasing the entanglement fidelity and rate [21]. Finally, errors arising from small inhomogeneities in the coupling strength can be suppressed with additional electron spin control [42].

Figure 4 shows the controlled-NOT gate error $\mathcal{E}_{T}$ as a function of $C$. For realistic parameters described in [42], at very high $C$, the total error $\mathcal{E}_{T}$ is limited by the electronnuclear two qubit gate fidelity, while at more modest cooperativity, the error scales favorably compared to the existing state of the art [17]. Note that an experimental demonstration of our protocol (with $\mathcal{E}_{T} \sim 10^{-1}$ ) may be

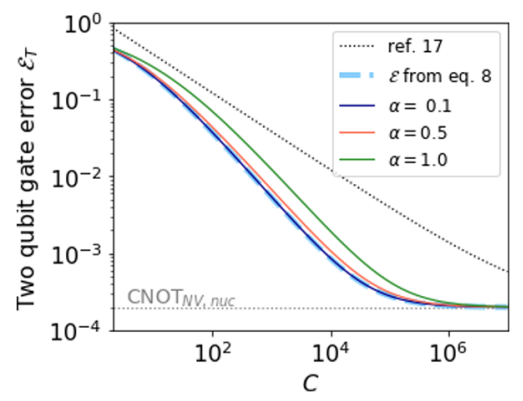

FIG. 4. Error $\mathcal{E}_{T}$ for teleported CNOT gate between two ${ }^{13} \mathrm{C}$ (or ${ }^{15} \mathrm{~N}$ ) nuclear spins as a function of $C$ for thresholds $\alpha$ and under Eq. (6). A mechanical NV spin readout using an interaction time of $20 t^{*}$ is assumed. The CNOT gate error between the NV and nuclear spin spin is set to $10^{-4}$ (dashed gray line) [73,74] and is also included in the representation of state-of-the-art hot gates [17]. Gate errors are numerically calculated (solid lines) and analytically approximated as $\mathcal{E}$ [Eq. (8), neglecting nuclear spin decoherence, dashed line]. For details, see text. 
possible at room temperature and $C \sim 8$, corresponding to state-of-the-art spin-mechanical systems $(1 / \Gamma \sim 10 \mathrm{~ms}$ [70]; $Q \sim 10^{9} \quad[9,71] ; \lambda / 2 \pi \sim 0.9 \mathrm{kHz}[41] ; \Delta m^{2} \sim 27$ [40]). These parameters are within reach for a soft-clamped, silicon nitride nanobeam resonator, functionalized with a nanomagnet at the antinode of motion and placed adjacent to diamond hosting NV centers [Fig. 1(d)] [70,71]. A modest further improvement in the spin-mechanical coupling strength $(\lambda \sim 2 \mathrm{kHz})$ enables high-fidelity gates $\left(\mathcal{E}_{T}<10^{-2}\right)$. Conversely, at cryogenic temperatures ( $T \sim 4 \mathrm{~K}$, coherence time $1 / \Gamma \sim 1 \mathrm{~s}[$ [70,72]) the same system can achieve this fidelity already with $\lambda / 2 \pi \sim$ $100 \mathrm{~Hz}$ [42]. Such parameters yield a high probability of success (approaching 50\% per run) and an average gate duration approaching $10 \mathrm{~ms}$, faster or comparable to deterministic protocols with the reported coupling strengths [17].

Conclusion.-We proposed and analyzed a half-parity measurement protocol in a decoherence free subspace for entangling two qubits through a hot resonator, with error scaling that nears $\mathcal{E} \propto \ln (C) / C$. Our protocol is fast and robust to thermalization errors, and it does not require ground state cooling. A teleported controlled-NOT gate employing the generated Bell pair is feasible with magnetically functionalized resonators and solid-state electronic spins featuring long coherence times [70,71]. While we analyzed an implementation involving NV centers, the protocol can also be applied to other promising paramagnetic defects, such as spins in silicon [75], as readout and initialization can be realized mechanically [11]. Further directions for analysis include leveraging continuous feedback to increase the entanglement rate $[21,22,76]$ and the application of our protocol to generate multipartite entangled states. Finally, for further improvements, nanobeam resonators can be electrostatically coupled [8] using hybridized mechanical modes to selectively couple spins adjacent to distinct resonators, enabling multiqubit connectivity far beyond the reach of the magnetic dipole-dipole interactions. While our work leverages decades of development of micromechanical devices and solid-state qubits, it simultaneously eliminates the need for high-fidelity single qubit optical or electronic addressing. With substantial technical improvement beyond the current state of the art, in the long term this approach could pave the way for realization of solid-state, room-temperature quantum information systems.

We would like to thank S. Hofer for insightful discussions and Samantha Dakoulas for assistance during this project. The authors thank C. Maxwell for design assistance with Figs. 1 and 2. This work was supported by NSF, CUA, ARO MURI, and V. Bush Faculty Fellowship. R. R. was supported by the Alexander von Humboldt Foundation. This material is based upon work supported by the National Science Foundation Graduate Research Fellowship under Grants No. DGE1144152 and
No. DGE1745303. This work was supported by DOE, Grant No. DE-SC0020115. Any opinion, findings, and conclusions or recommendations expressed in this material are those of the authors and do not necessarily reflect the views of the National Science Foundation.

*Corresponding author.

emma.louise.rosenfeld@gmail.com, she/her/hers

Present address: IAV GmbH DigitalLab.

†Previous address: Physics Department, Harvard University. This work was done prior to joining AWS.

[1] E. D. Herbschleb, H. Kato, Y. Maruyama, T. Danjo, T. Makino, S. Yamasaki, I. Ohki, K. Hayashi, H. Morishita, M. Fujiwara, and N. Mizuochi, Ultra-long coherence times amongst room-temperature solid-state spins, Nat. Commun. 10, 3766 (2019).

[2] F. Dolde, I. Jakobi, B. Naydenov, N. Zhao, S. Pezzagna, C. Trautmann, J. Meijer, P. Neumann, F. Jelezko, and J. Wrachtrup, Room-temperature entanglement between single defect spins in diamond, Nat. Phys. 9, 139 (2013).

[3] H. Bernien, B. Hensen, W. Pfaff, G. Koolstra, M. S. Blok, L. Robledo, T. H. Taminiau, M. Markham, D. J. Twitchen, L. Childress, and R. Hanson, Heralded entanglement between solid-state qubits separated by three metres, Nature (London) 497, 86 (2013).

[4] P. C. Humphreys, N. Kalb, J. P. J. Morits, R. N. Schouten, R. F. L. Vermeulen, D. J. Twitchen, M. Markham, and R. Hanson, Deterministic delivery of remote entanglement on a quantum network, Nature (London) 558, 268 (2018).

[5] J. Borregaard, P. Kómár, E. M. Kessler, A. S. Sørensen, and M. D. Lukin, Heralded Quantum Gates with Integrated Error Detection in Optical Cavities, Phys. Rev. Lett. 114, 110502 (2015).

[6] L. Jiang, M. V. Gurudev Dutt, E. Togan, L. Childress, P. Cappellaro, J. M. Taylor, and M. D. Lukin, Coherence of an Optically Illuminated Single Nuclear Spin Qubit, Phys. Rev. Lett. 100, 073001 (2008).

[7] N. Kalb, P. C. Humphreys, J. J. Slim, and R. Hanson, Dephasing mechanisms of diamond-based nuclear-spin memories for quantum networks, Phys. Rev. A 97, 062330 (2018).

[8] P. Rabl, S. J. Kolkowitz, F. H. L. Koppens, J. G. E. Harris, P. Zoller, and M. D. Lukin, A quantum spin transducer based on nanoelectromechanical resonator arrays, Nat. Phys. 6, 602 (2010).

[9] Y. Tsaturyan, A. Barg, E. S. Polzik, and A. Schliesser, Ultracoherent nanomechanical resonators via soft clamping and dissipation dilution, Nat. Nanotechnol. 12, 776 (2017).

[10] G. S. MacCabe, H. Ren, J. Luo, J. D. Cohen, H. Zhou, A. Sipahigil, M. Mirhosseini, and O. Painter, Nano-acoustic resonator with ultralong phonon lifetime, Science 370, 840 (2020)

[11] D. Rugar, R. Budakian, H. J. Mamin, and B. W. Chui, Single spin detection by magnetic resonance force microscopy, Nature (London) 430, 329 (2004).

[12] S. Hong, M. S. Grinolds, P. Maletinsky, R. L. Walsworth, M. D. Lukin, and A. Yacoby, Coherent, Mechanical Control of a Single Electronic Spin, Nano Lett. 12, 3920 (2012). 
[13] T. Delord, P. Huillery, L. Nicolas, and G. Hétet, Spincooling of the motion of a trapped diamond, Nature (London) 580, 56 (2020).

[14] D. Lee, K. W. Lee, J. V. Cady, P. Ovartchaiyapong, and A. C. Bleszynski Jayich, Topical review: spins and mechanics in diamond, J. Opt. 19, 033001 (2017).

[15] D. D. Bhaktavatsala Rao, S. Ali Momenzadeh, and J. Wrachtrup, Heralded Control of Mechanical Motion by Single Spins, Phys. Rev. Lett. 117, 077203 (2016).

[16] A. Sørensen and K. Mølmer, Quantum Computation with Ions in Thermal Motion, Phys. Rev. Lett. 82, 1971 (1999).

[17] M. J. A. Schuetz, G. Giedke, L. M. K. Vandersypen, and J. I. Cirac, High-fidelity hot gates for generic spin-resonator systems, Phys. Rev. A 95, 052335 (2017).

[18] A.S. Sørensen and K. Mølmer, Measurement Induced Entanglement and Quantum Computation with Atoms in Optical Cavities, Phys. Rev. Lett. 91, 097905 (2003).

[19] C. Hill and J. Ralph, Weak measurement and control of entanglement generation, Phys. Rev. A 77, 014305 (2008).

[20] N. Roch, M. E. Schwartz, F. Motzoi, C. Macklin, R. Vijay, A. W. Eddins, A. N. Korotkov, K. B. Whaley, M. Sarovar, and I. Siddiqi, Observation of Measurement-Induced Entanglement and Quantum Trajectories of Remote Superconducting Qubits, Phys. Rev. Lett. 112, 170501 (2014).

[21] L. Martin, F. Motzoi, H. Li, M. Sarovar, and B. Whaley, Deterministic generation of remote entanglement with active quantum feedback, Phys. Rev. A 92, 062321 (2015).

[22] L. Martin, M. Sayrafi, and K. B. Whaley, What is the optimal way to prepare a Bell state using measurement and feedback?, Quantum Sci. Technol. 2, 044006 (2017).

[23] S. Kolkowitz, A.C. Bleszynski Jayich, Q.P. Unterreithmeier, S. D. Bennett, P. Rabl, J. G. E. Harris, and M. D. Lukin, Coherent Sensing of a Mechanical Resonator with a Single-Spin Qubit, Science 335, 1603 (2012).

[24] D. J. Christle, A. L. Falk, P. Andrich, P. V. Klimov, J. Ul Hassan, N. T. Son, E. Janzén, T. Ohshima, and D. D. Awschalom, Isolated electron spins in silicon carbide with millisecond coherence times, Nat. Mater. 14, 160 (2015).

[25] S. Ali Momenzadeh, F. Fávaro de Oliveira, P. Neumann, D. D. B. Rao, A. Denisenko, M. Amjadi, Z. Chu, S. Yang, N. B. Manson, M. W. Doherty, and J. Wrachtrup, Thin Circular Diamond Membrane with Embedded NitrogenVacancy Centers for Hybrid Spin-Mechanical Quantum Systems, Phys. Rev. Applied 6, 024026 (2016).

[26] A. Barfuss, J. Teissier, E. Neu, A. Nunnenkamp, and P. Maletinsky, Strong mechanical driving of a single electron spin, Nat. Phys. 11, 820 (2015).

[27] M. S. J. Barson, P. Peddibhotla, P. Ovartchaiyapong, K. Ganesan, R. L. Taylor, M. Gebert, Z. Mielens, B. Koslowski, D. A. Simpson, L. P. McGuinness, J. McCallum, S. Prawer, S. Onoda, T. Ohshima, A. C. Bleszynski Jayich, F. Jelezko, N. B. Manson, and M. W. Doherty, Nanomechanical Sensing Using Spins in Diamond, Nano Lett. 17, 1496 (2017).

[28] D. Andrew Golter, T. Oo, M. Amezcua, K. A. Stewart, and H. Wang, Optomechanical Quantum Control of a NitrogenVacancy Center in Diamond, Phys. Rev. Lett. 116, 143602 (2016).

[29] B. Khanaliloo, H. Jayakumar, A. C. Hryciw, D. P. Lake, H. Kaviani, and P.E. Barclay, Single-Crystal Diamond
Nanobeam Waveguide Optomechanics, Phys. Rev. X 5, 041051 (2015).

[30] E. R. MacQuarrie, T. A. Gosavi, N. R. Jungwirth, S. A. Bhave, and G. D. Fuchs, Mechanical Spin Control of Nitrogen-Vacancy Centers in Diamond, Phys. Rev. Lett. 111, 227602 (2013).

[31] S. Meesala, Y.-I. Sohn, H. A. Atikian, S. Kim, M. J. Burek, J. T. Choy, and M. Lončar, Enhanced Strain Coupling of Nitrogen-Vacancy Spins to Nanoscale Diamond Cantilevers, Phys. Rev. Applied 5, 034010 (2016).

[32] P. Ovartchaiyapong, K. W. Lee, B. A. Myers, and A. C. Bleszynski Jayich, Dynamic strain-mediated coupling of a single diamond spin to a mechanical resonator, Nat. Commun. 5, 4429 (2014).

[33] J. Teissier, A. Barfuss, P. Appel, E. Neu, and P. Maletinsky, Strain Coupling of a Nitrogen-Vacancy Center Spin to a Diamond Mechanical Oscillator, Phys. Rev. Lett. 113, 020503 (2014).

[34] J. Gieseler, A. Kabcenell, E. Rosenfeld, J. D. Schaefer, A. Safira, M. J. A. Schuetz, C. Gonzalez-Ballestero, C. C. Rusconi, O. Romero-Isart, and M. D. Lukin, Single-Spin Magnetomechanics with Levitated Micromagnets, Phys. Rev. Lett. 124, 163604 (2020).

[35] J. G. Longenecker, H. J. Mamin, A. W. Senko, L. Chen, C. T. Rettner, D. Rugar, and J. A. Marohn, High-Gradient Nanomagnets on Cantilevers for Sensitive Detection of Nuclear Magnetic Resonance, ACS Nano 6, 9637 (2012).

[36] J. M. Nichol, E. R. Hemesath, L. J. Lauhon, and R. Budakian, Nanomechanical detection of nuclear magnetic resonance using a silicon nanowire oscillator, Phys. Rev. B 85, 054414 (2012).

[37] R. Fischer, D. P. McNally, C. Reetz, G. G. T. Assumpção, T. Knief, Y. Lin, and C. A. Regal, Spin detection with a micromechanical trampoline: Towards magnetic resonance microscopy harnessing cavity optomechanics, New J. Phys. 21, 043049 (2019).

[38] M. Rossi, D. Mason, J. Chen, Y. Tsaturyan, and A. Schliesser, Measurement-based quantum control of mechanical motion, Nature (London) 563, 53 (2018).

[39] M. H. Abobeih, J. Cramer, M. A. Bakker, N. Kalb, M. Markham, D. J. Twitchen, and T. H. Taminiau, One-second coherence for a single electron spin coupled to a multiqubit nuclear-spin environment, Nat. Commun. 9, 2552 (2018).

[40] J. Guo, R. Norte, and S. Gröblacher, Feedback Cooling of a Room Temperature Mechanical Oscillator close to its Motional Ground State, Phys. Rev. Lett. 123, 223602 (2019).

[41] O. Arcizet, V. Jacques, A. Siria, P. Poncharal, P. Vincent, and S. Seidelin, A single nitrogen-vacancy defect coupled to a nanomechanical oscillator, Nat. Phys. 7, 879 (2011).

[42] See Supplemental Material at http://link.aps.org/supplemental/ 10.1103/PhysRevLett.126.250505 for further details including, but not limited to, equations of motion, effects from inhomogeneous coupling strength and proposed system parameters.

[43] C. E. Bradley, J. Randall, M. H. Abobeih, R. C. Berrevoets, M. J. Degen, M. A. Bakker, M. Markham, D. J. Twitchen, and T. H. Taminiau, A Ten-Qubit Solid-State Spin Register with Quantum Memory up to One Minute, Phys. Rev. X 9 , 031045 (2019). 
[44] C. T. Nguyen, D. D. Sukachev, M. K. Bhaskar, B. Machielse, D. S. Levonian, E. N. Knall, P. Stroganov, C. Chia, M. J. Burek, R. Riedinger, H. Park, M. Lončar, and M. D. Lukin, An integrated nanophotonic quantum register based on silicon-vacancy spins in diamond, Phys. Rev. B 100, 165428 (2019).

[45] G. Waldherr, Y. Wang, S. Zaiser, M. Jamali, T. SchulteHerbrüggen, H. Abe, T. Ohshima, J. Isoya, J. F. Du, P. Neumann, and J. Wrachtrup, Quantum error correction in a solid-state hybrid spin register, Nature (London) 506, 204 (2014).

[46] T. van der Sar, Z. H. Wang, M. S. Blok, H. Bernien, T. H. Taminiau, D. M. Toyli, D. A. Lidar, D. D. Awschalom, R. Hanson, and V. V. Dobrovitski, Decoherence-protected quantum gates for a hybrid solid-state spin register, Nature (London) 484, 82 (2012).

[47] L. Jiang, J. S. Hodges, J. R. Maze, P. Maurer, J. M. Taylor, D. G. Cory, P. R. Hemmer, R. L. Walsworth, A. Yacoby, A. S. Zibrov, and M. D. Lukin, Repetitive readout of a single electronic spin via quantum logic with nuclear spin ancillae, Science 326, 267 (2009).

[48] G. Liu, M. Chen, Y.-X. Liu, D. Layden, and P. Cappellaro, Repetitive readout enhanced by machine learning, Mach. Learn. Sci. Technol. 1, 015003 (2020).

[49] Y. Doi, T. Makino, H. Kato, D. Takeuchi, M. Ogura, H. Okushi, H. Morishita, T. Tashima, S. Miwa, S. Yamasaki, P. Neumann, J. Wrachtrup, Y. Suzuki, and N. Mizuochi, Deterministic Electrical Charge-State Initialization of Single Nitrogen-Vacancy Center in Diamond, Phys. Rev. X 4, 011057 (2014).

[50] Y. Doi, T. Fukui, H. Kato, T. Makino, S. Yamasaki, T. Tashima, H. Morishita, S. Miwa, F. Jelezko, Y. Suzuki, and N. Mizuochi, Pure negatively charged state of the NV center in $\$ n$ \$-type diamond, Phys. Rev. B 93, 081203(R) (2016).

[51] A. Dréau, P. Spinicelli, J. R. Maze, J.-F. Roch, and V. Jacques, Single-Shot Readout of Multiple Nuclear Spin Qubits in Diamond under Ambient Conditions, Phys. Rev. Lett. 110, 060502 (2013).

[52] M. Chiani, D. Dardari, and M. K. Simon, New exponential bounds and approximations for the computation of error probability in fading channels, IEEE Trans. Wireless Commun. 2, 840 (2003).

[53] D. A. Hopper, J. D. Lauigan, T.-Y. Huang, and L. C. Bassett, Real-Time Charge Initialization of Diamond NitrogenVacancy Centers for Enhanced Spin Readout, Phys. Rev. Applied 13, 024016 (2020).

[54] M. R. Vanner, I. Pikovski, G. D. Cole, M. S. Kim, C. Brukner, K. Hammerer, G. J. Milburn, and M. Aspelmeyer, Pulsed quantum optomechanics, Proc. Natl. Acad. Sci. U.S.A. 108, 16182 (2011).

[55] S. G. Hofer and K. Hammerer, Quantum Control of Optomechanical Systems, in Advances In Atomic, Molecular, and Optical Physics (Elsevier, New York, 2017), Vol. 66, pp. 263-374, https://www.sciencedirect.com/science/article/ pii/S1049250X17300149.

[56] V. Hassani, A. P. Aguiar, A. M. Pascoal, and M. Athans, Further results on plant parameter identification using continuous-time multiple-model adaptive estimators, in Proceedings of the 48h IEEE Conference on Decision and Control (CDC) Held Jointly with 2009 28th Chinese
Control Conference (2009), pp. 7261-7266, https:// ieeexplore.ieee.org/document/5400434.

[57] R. F. Stengel, Optimal Control and Estimation, reissue edition (Dover Publications, New York, 1994).

[58] A. E. Bryson, Jr. and Y.-C. Ho, Applied Optimal Control: Optimization, Estimation and Control, 1st ed. (CRC Press, New York, 1975).

[59] K. S. Chou, J. Z. Blumoff, C. S. Wang, P. C. Reinhold, C. J. Axline, Y. Y. Gao, L. Frunzio, M. H. Devoret, L. Jiang, and R. J. Schoelkopf, Deterministic teleportation of a quantum gate between two logical qubits, Nature (London) 561, 368 (2018).

[60] X. Rong, J. Geng, F. Shi, Y. Liu, K. Xu, W. Ma, F. Kong, Z. Jiang, Y. Wu, and J. Du, Experimental fault-tolerant universal quantum gates with solid-state spins under ambient conditions, Nat. Commun. 6, 8748 (2015).

[61] T. P. Harty, D. T. C. Allcock, C. J. Ballance, L. Guidoni, H. A. Janacek, N. M. Linke, D. N. Stacey, and D. M. Lucas, High-Fidelity Preparation, Gates, Memory, and Readout of a Trapped-Ion Quantum Bit, Phys. Rev. Lett. 113, 220501 (2014).

[62] G. T. Genov, D. Schraft, N. V. Vitanov, and T. Halfmann, Arbitrarily Accurate Pulse Sequences for Robust Dynamical Decoupling, Phys. Rev. Lett. 118, 133202 (2017).

[63] A. Reiserer, N. Kalb, M. S. Blok, K. J. M. van Bemmelen, T. H. Taminiau, R. Hanson, D. J. Twitchen, and M. Markham, Robust Quantum-Network Memory Using Decoherence-Protected Subspaces of Nuclear Spins, Phys. Rev. X 6, 021040 (2016).

[64] D. A. Hopper, H. J. Shulevitz, and L. C. Bassett, Spin Readout Techniques of the Nitrogen-Vacancy Center in Diamond, Micromachines 9, 437 (2018).

[65] M. A. Perlin, Z.-Y. Wang, J. Casanova, and M. B. Plenio, Noise-resilient architecture of a hybrid electron-nuclear quantum register in diamond, Quantum Sci. Technol. 4, 015007 (2018).

[66] G. P. Berman, D. I. Kamenev, and V. I. Tsifrinovich, Stationary cantilever vibrations in oscillating-cantilever-driven adiabatic reversals: Magnetic-resonance-force-microscopy technique, Phys. Rev. A 66, 023405 (2002).

[67] W. Wieczorek, S. G. Hofer, J. Hoelscher-Obermaier, R. Riedinger, K. Hammerer, and M. Aspelmeyer, Optimal State Estimation for Cavity Optomechanical Systems, Phys. Rev. Lett. 114, 223601 (2015).

[68] A. P. Aguiar, M. Athans, and A. M. Pascoal, Convergence properties of a Continuous-Time Multiple-Model Adaptive Estimator, in 2007 European Control Conference (ECC) (2007), pp. 1530-1536, https://ieeexplore.ieee.org/ document/7068291.

[69] P. D. Hanlon and P.S. Maybeck, Multiple-model adaptive estimation using a residual correlation Kalman filter bank, IEEE Trans. Aerospace Electron. Syst. 36, 393 (2000).

[70] N. Bar-Gill, L. M. Pham, A. Jarmola, D. Budker, and R. L. Walsworth, Solid-state electronic spin coherence time approaching one second, Nat. Commun. 4, 1743 (2013).

[71] A. H. Ghadimi, S. A. Fedorov, N. J. Engelsen, M. J. Bereyhi, R. Schilling, D. J. Wilson, and T. J. Kippenberg, Elastic strain engineering for ultralow mechanical dissipation, Science 360, 764 (2018).

[72] M. H. Abobeih, J. Randall, C. E. Bradley, H. P. Bartling, M. A. Bakker, M. J. Degen, M. Markham, D. J. Twitchen, and 
T. H. Taminiau, Atomic-scale imaging of a 27-nuclear-spin cluster using a quantum sensor, Nature (London) 576, 411 (2019).

[73] Y. Chou, S.-Y. Huang, and H.-S. Goan, Optimal control of fast and high-fidelity quantum gates with electron and nuclear spins of a nitrogen-vacancy center in diamond, Phys. Rev. A 91, 052315 (2015).

[74] W. Dong, F. A. Calderon-Vargas, and S. E. Economou, Precise high-fidelity electron-nuclear spin entangling gates in NV centers via hybrid dynamical decoupling sequences, New J. Phys. 22, 073059 (2020).

[75] J. J. Pla, K. Y. Tan, J. P. Dehollain, W. H. Lim, J. J. L. Morton, D. N. Jamieson, A. S. Dzurak, and A. Morello, A single-atom electron spin qubit in silicon, Nature (London) 489, 541 (2012).

[76] L.S. Martin and K. B. Whaley, Single-shot deterministic entanglement between non-interacting systems with linear optics, arXiv:1912.00067. 